\title{
The international journal of material forming - 10th anniversary
}

\author{
Francisco Chinesta $^{1}$
}

Published online: 13 January 2021

(C) The Author(s), under exclusive licence to Springer-Verlag France SAS part of Springer Nature 2021

The International Journal of Material Forming -IJMF- was celebrating its 10th anniversary in 2018. With its first issue published in 2008, IJMF continues being a key figure in the science and technology of material forming, covering different approaches (modeling, simulation and experiments), materials (including composites) and forming processes.

The journal, published by Springer, was from its origin, the official journal of the ESAFORM association, and both are progressing and adapting to the evolving environment in very close connection.

For celebrating its 10th anniversary two topical issues were launched. The second one, the present, covers the broad topic of composites and more particularly, it aims at bridging scales as well as chaining from the materials to the component or structure performances.

The influence of the manufacturing operations on the final performance of the composites products has been acknowledged since the very beginning of the history of composite materials. The difficulty to predict and control this influence was out of reach until recently and therefore the engineers developed approaches based heavily on knock-down factors. After years of pioneering works, one can observe that the accounting of the fiber orientations after forming is slowly becoming the best practice in industry today. However, beyond this first order parameter, the best-practice remains the use of predefined quality criteria and fixed manufacturing process window to define the quality of a composite structure. The global status could be described as a frozen status with limited room for change.

In parallel, the changes introduced in manufacturing for a few years by a massive and rapid transition toward a complete digitalization of industry created an unthinkable wealth of new opportunities. By smart use of information coming from sensors during production, it seems that what was out of control

Francisco Chinesta

Francisco.CHINESTA@ensam.eu

1 ENSAM ParisTech, Paris, France becomes magically under control. There is indeed no discussion about the incredible amount of events that can be now monitored during the actual manufacturing from the constituent materials (resin, fibers, preforms, prepregs) to the various equipment along a production line together with the various and improved quality controls. However, it would be a terrible mistake to decide stopping the effort launched by the community in the modeling of the effects of composites manufacturing onto the mechanical performance. It would be even worse than having stopped the study of materials when we moved from the use of water steam energy to electricity. At the opposite, the current revolutionary technological progresses in manufacturing need a strong physical modeling of the composite materials across each and every manufacturing steps and life phases till recycling to enable the full delivery of the promises of the running evolution. Being able to model with physics each manufacturing step, using the outcome of the former steps and providing information to the next step is key to fully exploit the support issued by the sensors: the larger the scientific knowledge, the more meaningful the data become.

Our new world will emerge from a smart combination of the traditional science and of the machines empowered by artificial intelligence. This is especially true with composite materials and this special issue on the effects of manufacturing on mechanical performance is a significant contribution to the movement.

With this issue, the so-called digital thread supporting the exponential natality of digital twins will find examples of 'physics-based' tools to be plugged in. It is also a call to proceed with the effort because data need physics.

In this issue you will find then some works for instance on the effects of local fiber deformation like shear and waviness but also of typical consequences of manufacturing like porosities on the mechanical properties and performance - with a special focus on the AFP process. The drapeability remains an active topic of research and due to the first order impact on properties, this is a good thing. Complex physics involving electromagnetism is also tackled, and finally a PGD 
application is presented opening the door for real time applications in factories.

Prof. Anais Barasinski,

Dr. Patrick de Luca,

Prof. Kiyoshi Uzawa,

Prof. Anthony M. Waas

Invited Editors
Francisco Chinesta

Editor in Chief of IJMF

Publisher's note Springer Nature remains neutral with regard to jurisdictional claims in published maps and institutional affiliations. 